\title{
Régie et archivage au quotidien des représentations
}

Daily Management and Archiving of Performances

Daniel Deshays

\section{(2) OpenEdition}

Journals

Édition électronique

URL : https://journals.openedition.org/rsl/1829

DOI : $10.4000 /$ rsl. 1829

ISSN : 2271-6246

Éditeur

Éditions Rue d'Ulm

Référence électronique

Daniel Deshays, «Régie et archivage au quotidien des représentations », Revue Sciences/Lettres [En ligne], 6 | 2019, mis en ligne le 10 décembre 2018, consulté le 29 juillet 2021. URL : http:// journals.openedition.org/rsl/1829; DOI : https://doi.org/10.4000/rsl.1829

Ce document a été généré automatiquement le 29 juillet 2021.

(c) Revue Sciences/Lettres 


\title{
Régie et archivage au quotidien des représentations
}

\author{
Daily Management and Archiving of Performances
}

Daniel Deshays

À mes amis les régisseurs du son.

1 Le sujet n'étant pas encore véritablement exploré, il faut mettre au conditionnel l'état des lieux que j'ébauche ici pour les années 1947-1997. Les entretiens que j'ai réalisés auprès de témoins actifs du son radiophonique et du spectacle vivant de cette époque ne sont pas assez nombreux pour rendre compte de la diversité des pratiques professionnelles d'alors. Toutefois, l'arrivée des nouveaux outils et l'apparition de nouvelles fonctions de régie du son n'ayant pas engendré d'approches très variées, nous pouvons considérer, au regard de ma propre mémoire des pratiques des années 1970, que ces témoignages fournissent un matériau - rare -, qui peut être considéré comme référent.

\section{Préambule}

2 La spécificité du sonore, ce médium du vivant, n'est pas bien comprise. Pour replacer le sonore du théâtre à sa juste place face à la voix, je dirais que le sonore diffusé a quasiment la même existence - en raison de sa nature temporelle et énergétique - que le sonore issu du vivant mis en scène : voix et mouvements des corps des acteurs. De ce point de vue - consistant à prendre en compte la nature du son -, la voix égale n'importe quel son, et inversement. Les scénographies, les costumes et même l'éclairage, qui tentent de construire le temps, ne peuvent se tenir à cette place propre au son: appartenir au monde des objets éphémères et fragiles. Le son doit rendre compte des matérialités en transformation, dans la précision des mouvements, dans la qualité fugitive des expressions; une fragilité, apanage du vivant, que la régie du son doit faire perdurer depuis les canaux électroacoustiques de l'amplification. À ce titre, il y a bien égalité des conditions d'existence de l'ensemble des états du son: tout y exprime la nature des relations qui se jouent ; à ce titre la condition d'existence de la 
voix n'est en rien différente de celle des autres sons. Ceci est à mettre au bénéfice du travail des régisseurs son et des concepteurs, bien sûr.

\section{Invisible régie}

3 Les travaux artistiques - car il s'agit bien d'un art - de ceux qui, chaque soir, ont régi le son, la lumière et le plateau des théâtres nous demeurent parfaitement inconnus. Ils rendent pourtant compte de l'évolution historique du théâtre. La mémoire que les techniciens nous livrent encore, et dont j'ébauche ici la transmission, permet de mieux comprendre ce que l'archive recèle en termes d'évolution des modalités de son inscription sur un support.

Comme je l'indiquais dans un texte récemment $\operatorname{paru}^{1}$, les régies lumière et son des théâtres ont migré de la scène vers le fond de la salle durant la période 1960-1970 pour des raisons de surcharge d'activités assemblées au plateau, devenues impossibles à gérer. Jusqu'alors, le plus souvent, le régisseur général veillait en scène à la machinerie, mais aussi aux régies de la lumière et du son; il rédigeait, lors des répétitions, le cahier de régie. L'accélération de l'emploi du son est due à l'arrivée des nouveaux supports, les "matières plastiques", comme on disait: le vinyle pour les disques, l'acétate puis le polyester pour les bandes magnétiques, autorisaient des usages multipliés. La création des cabines de régie a en effet coïncidé avec l'arrivée des magnétophones qui remplacèrent les phonographes.

5 Les développements technologiques qui ont surgi au début des années soixante avec l'émergence des musiques amplifiées ont eux aussi changé la donne. Du fait de l'augmentation de leur nombre, les concerts de variétés, faute de salles disponibles et suffisamment grandes, ont régulièrement investi les plateaux des théâtres. En découvrant ces musiques, la période devait surtout inventer les moyens de les faire entendre. Et le spectacle des nouvelles musiques portait avec lui le spectacle de sa technologie. La vue des murs d'enceintes, offerts à tous sur les scènes de la pop, icônes de la puissance qui s'y exprimait, ne manqua pas d'aiguiser les envies. Ceux qui s'autoproclamaient techniciens du spectacle, mais aussi les comédiens et les metteurs en scène eux-mêmes, élaboraient des projets instruits de cette vision. Ce certificat de nouveauté du théâtre validé par l'usage des avancées technologiques n'a toujours pas quitté les plateaux. L'emploi d'une « technologie nouvelle ${ }^{2}$ », formule magique, serait la garantie de l'actualisation du propos théâtral.

6 Au théâtre, jusqu'au début des années 1950, l'éclairage était réglé par le metteur en scène lui-même. C'est Jean Vilar qui décida de déléguer à l'un de ses électriciens, Pierre Saveron, la fonction d'éclairagiste. Pour autant, la naissance de cette nouvelle fonction n'induisait pas forcément la suivante et le régisseur son attendit encore avant d'apparaître. Ce fut un comédien ami de Vilar, Maurice Coussonneau, qui régit le son à Chaillot jusqu'en 1963, date de reprise de la direction par Georges Wilson. Sa fonction se limitait essentiellement à « envoyer les musiques ». Avant les années 1970, aucune école ne formait sérieusement les régisseurs, tout au plus existait-il à l'école de la rue Blanche quelques visites aux théâtres et des cours sur table. Il fallut encore quelques années pour qu'une véritable formation apparaisse ${ }^{3}$.

7 Les théâtres qui devaient s'équiper en matériel d'éclairage ajournaient encore un peu plus leurs acquisitions du côté du son. Le matériel, parfois acheté neuf, devait être 
polyvalent (musique-théâtre), de sorte qu'il ne convenait vraiment à aucun des domaines, trop faible pour la musique, trop puissant pour s'accorder à l'échelle de la voix parlée. S'il est difficile de faire un état des lieux des équipements sonores des théâtres d'alors, on peut se dire que les choix n'étaient pas si divers, car peu de constructeurs existaient. Il faut cependant rappeler que si la programmation des salles a toujours eu des formes variées, entraînant une nécessité de polyvalence du matériel, la diffusion sonore, quant à elle, nécessite un matériel spécifique pour chaque domaine.

\section{Le régisseur}

8 Les régisseurs circulaient peu au début, le statut d'intermittent créé en 1936 pour le cinéma ne devant entrer en vigueur qu'en janvier 1968 pour le spectacle vivant. Appartenant à des établissements distincts, les techniciens se rencontraient rarement, mais pour autant, les pratiques ne divergeaient guère. Le poste de régisseur son et lumière était dévolu aux personnes intéressées par l'électricité, lesquelles n'avaient généralement qu'une connaissance sommaire des secteurs artistiques dans lesquels elles évoluaient. Les théâtres municipaux ou privés engageaient des régisseurs sans spécialité et c'est en interne que la distribution sur les postes s'effectuait. L'apprentissage s'affûtait plus ou moins dans l'expérience des répétitions et des spectacles. C'est seul ou à deux que ces techniciens régissaient la lumière et le son, dans une cabine étroite et parfois aveugle. La régie s'y effectuait à l'oreille, la scène était entendue à travers des haut-parleurs souvent de médiocre qualité alimentés par un système de prise de son constitué de deux micros situés le plus souvent à proximité du cadre de scène. Signalons dès maintenant que ce sont ces mêmes micros qui alimentaient le magnétophone chargé d'enregistrer le spectacle en vue de son archivage. Ce sont donc les bandes issues de ces prises de son, des prises effectuées dans l'aléa d'une position microphonique souvent définie de la même manière, quel que soit le spectacle, que l'on retrouve dans les archives.

9 Les équipements de régie étaient le plus souvent choisis en relation au catalogue des fournisseurs auxquels les théâtres étaient liés. C'est souvent l'accord budgétaire établi par le directeur technique avec un distributeur de matériel qui déterminait les quelques achats technologiques. Par conséquent, les outils commandés n'étaient pas forcément les plus adaptés à tous les types de théâtre produits. Il faut rappeler que si les choix étaient effectués préférentiellement pour un usage musical, c'est le «Public Address ", c'est-à-dire l'amplification des réseaux d'ordre du théâtre, qui orientait le deuxième choix ${ }^{4}$. Ainsi les maisons Bouyer ou Philips équipaient des églises, des gares et certains théatres, depuis les halls et les couloirs jusqu'à la scène, avec des systèmes vissés au cadre et, de fait, impossibles à déplacer. Le déficit de compétence de certains régisseurs a induit également des choix de consoles, enregistreurs et micros pas ou peu adaptés aux contraintes quotidiennes. C'est le bouche-à-oreille ou la rencontre d'un régisseur de tournée qui pouvait réorienter un choix. Quoi qu'il en soit, le matériel adapté spécifiquement au théâtre était durant les années 1950-1970 particulièrement rare. Il l'est aujourd'hui à peine un peu moins, le théatre ne représentant toujours pas un marché rentable pour les fabricants.

10 Cet état de fait a perduré jusque dans les années 1990.

11 C'est la venue du numérique qui, après un temps d'attente prolongé jusqu'à l'arrivée de sa deuxième génération, a décidé la plupart des théâtres à reconsidérer leurs 
équipements. Ce ne fut pas pour autant immédiat et la qualité des outils numériques utilisés était loin de répondre à ce qui était attendu et d'équivaloir à l'analogique, qui se trouvait pourtant peu à peu abandonné. L'effet de mode et la persuasion des vendeurs faisaient accroire aux régisseurs que le nom même de numérique était un absolu, donc une garantie de qualité. La discorde régnait aussi entre les régisseurs et il fallut bien des années et des comparaisons tâtonnantes pour que, dans cet idéalisme, les avantages éventuels puissent être aperçus. Les offres se multipliaient et on pouvait échanger chez le revendeur, moyennant un peu d'argent, un Revox A77, voire un PR99, contre un lecteur Minidisc Sony qui prétendait rénover un parc vieillissant. De fait, l'électronique n'est pas éternelle et si les machines des studios étaient maintenues quotidiennement en état par des ingénieurs, les théâtres, pour d'évidentes raisons économiques, ne pouvaient engager une personne possédant ces compétences. La crainte principale était de tomber en panne en plein spectacle. C'est ainsi qu'attirés à la fois par la nouveauté et par la nécessité de renouveler un matériel obsolète qui tournait quotidiennement, les théâtres finirent par remettre leur sort entre les mains de sociétés qui leur vendaient ce qu'elles voulaient.

Seuls les grands théâtres ou ceux qui venaient de se construire se voyaient dotés d'équipements qui, en raison de leur budget, pouvaient atteindre une qualité «Broadcast ». Défini comme " professionnel », le Broadcast équipait les télévisions et les radios publiques. Loin de disposer des sommes nécessaires à une telle acquisition, la grande majorité des établissements utilisait des outils qui ne répondaient pas à ces normes. Si cette réalité n'était pas forcément perçue en direct par les spectateurs, nous la découvrons aujourd'hui en écoutant leurs archives, quand elles existent. Pour autant, cette répartition technique n'a pas un caractère absolu, des théâtres importants pouvaient aussi bien s'équiper de matériels dits de Hi-Fi, tant leur norme et leur qualité bien sélectionnées pouvaient parfois avoisiner le Broadcast.

Les enregistreurs $\mathrm{K} 7 \mathrm{Hi}-\mathrm{Fi}$ étaient équipés de réducteurs de bruit qui leur étaient spécifiques, Dolby B, C ou DBX. Les archives retrouvées dans ces théâtres, ne relevant donc pas de normes standardisées, sonnent parfois trop aiguës à la réécoute, en raison d'une absence de spécification sur les boîtes d'archivage ou de la disparition de certains des outils de décodage utilisés alors. Les enregistreurs pouvaient facilement être mal entretenus: têtes encrassées ou mal azimutées, faisant perdre des aigus dans les enregistrements. Il en résulte une diversité de résultats sonores qui entraînent de grandes différences dans la qualité des archives, y compris dans un même théâtre, selon les années ou selon les régisseurs.

\section{L'archivage des spectacles}

Nous écouterons successivement les voix et les témoignages de trois régisseurs : Albert Laracine, Raymond Burger et Didier Monfajon.

\section{État des lieux des pratiques de captation radiophonique du théâtre dans l'immédiat après-guerre}

15 Albert Laracine entre à la radio au lendemain de la Libération de Paris dans les services du Club d'Essai, rue de l'Université. Le groupe constitue un espace de recherche qui rassemble six élèves et quatre professionnels du son. Si la monophonie est encore la 
norme, très vite les recherches s'effectuent en direction de la mise au point de la stéréophonie. L'équipe pionnière inclut Albert Laracine, avec à ses côtés Jacques Chardonnier, puis Madeleine Sola et Daniel Toursière notamment. Dans un entretien que nous avons réalisé en collaboration avec Melissa van Drie en janvier 2015, Albert Laracine rend compte des méthodes utilisées pour les prises de son que la radio effectuait dans les théâtres parisiens en vue de leur diffusion sur les ondes.

Melissa van Drie : À l'écoute des enregistrements, nous essayons de comprendre comment les captations étaient faites.

Albert Laracine : La première que j'ai faite, c'était en 1946, c'était dans un théâtre des Grands Boulevards. Ce n'était pas la première, parce que dès qu'il y a eu des micros, la radio a retransmis des pièces. Ce que m'ont dit mes prédécesseurs, c'est que, tout bêtement, ils plaçaient un micro pendu au milieu de la scène. À mon époque on n'avait que trois micros : un micro était réservé pour le commentateur, car c'était retransmis en direct, et un micro était placé en scène. Alors il y avait deux cas : soit on le pendait au milieu de la scène, pour le théâtre classique, soit, pour les comédies, on l'avançait au-dessus de la rampe, dirigé vers les comédiens. Mais souvent les gens de théâtre ne voulaient pas que l'on pende des micros ; c'est la raison pour laquelle beaucoup privilégiaient les micros en rampe : deux micros posés au sol, au nez de scène - je ne dis pas que tout le monde faisait ainsi -, on les disposait assez distants car les comédiens se déplacent. Quand j'ai commencé, on enregistrait sur disque souple.

Daniel Deshays : Combien de temps durait l'enregistrement sur un disque souple?

$\mathrm{AL}:$ Ça durait trois minutes.

DD : Pour une pièce d'une heure trente, vous changiez toutes les trois minutes?

AL : Et ils étaient fragiles. Quand on les avait écoutés quatre ou cinq fois, c'était foutu. On gravait tout dessus : le théâtre, la musique, les interviews. Au théâtre, on avait deux platines d'enregistrement. On gravait la fin d'un disque avec le début de l'autre, et à la diffusion, avec un casque, on écoutait les deux et on faisait la commutation pour les synchroniser. Il y avait des pièces pour lesquelles on diffusait sans enregistrer, d'autres pour lesquelles on enregistrait et diffusait après, et parfois, on faisait les deux ensemble. Pour les concerts, c'était pareil.

MVD : Où était le lieu d'enregistrement, la cabine?

$\mathrm{AL}$ : On se mettait dans une loge de comédien, assez loin de la scène pour ne pas être gêné.

DD : C'était quels types de micros?

AL : C'était en principe des dynamiques ${ }^{5}$, soit des rubans Mélodium, des D021, ou des petits micros pas très bons, mais il y a vite eu des Neumann électrostatiques. Ils avaient une alimentation à piles, grosse comme une bouteille, c'était très bon mais ça avait l'ennui d'être gros. Et puis on a eu un petit micro Mélodium. Il y avait aussi des boules noires qui étaient des dynamiques ${ }^{6}$. On utilisait surtout les rubans, de bons micros confortables dans le grave, puis sont arrivés les micros électrostatiques Schoeps. Au labo on a fait plein d'essais avec la maison Schoeps, on travaillait régulièrement avec eux.

DD : Vous écoutiez sur quoi à l'époque? 
AL : Dans les théâtres on écoutait sur casque parce qu'on n'avait pas de place dans les loges. Quand on pouvait, on avait un ampli et deux haut-parleurs qu'on plaçait au mieux. Les enceintes, c'était n'importe quoi, des boîtes en bois avec des hautparleurs dedans. Mais tout le monde écoutait là-dessus. Les petits haut-parleurs des récepteurs du commerce n'étaient pas meilleurs.

Puis on a eu des consoles de prise de son avec plus de micros, on en a eu six en extérieur. On les suspendait autour de la scène, ça dépendait du jeu, on mettait des [micros] suspendus plus ou moins à la rampe, mais c'était toujours du mono. Quand il y avait un aparté on allait parfois placer un petit micro d'appoint mais on ne pouvait faire mieux.

Tout s'est compliqué à l'arrivée de la stéréophonie. C'était bien mieux, mais plus difficile : il fallait donner aux auditeurs la qualité du son, la qualité acoustique, mais aussi un espace sonore cohérent. On utilisait les couples stéréo qu'on avait mis au point dans les années cinquante; on pouvait en placer un en parapluie au-dessus de la scène, au milieu, mais les metteurs en scène ne supportaient pas qu'on voie les micros, alors on les plaçait à la rampe, mais ça obligeait le preneur de son à suivre les déplacements des acteurs de manière à ce qu'il n'y ait pas de distorsion d'espace. Évidemment quand on mettait un micro suspendu au centre ça se passait tout seul.

DD : Qu'est-ce que ça posait comme problèmes de les installer à la place du souffleur?

AL : On avait une perte de niveau sur les éloignements et c'était trop large. À la répétition on plaçait les micros et quelqu'un passait devant en parlant de manière à pouvoir les équilibrer et qu'on entende à peu près partout pareil.

DD : Vous enregistriez dans quels théâtres?

AL : Moi j'allais dans tous les théâtres de Paris et de la région parisienne. On se réinstallait à chaque fois. Il n'y avait que quelques salles qui étaient équipées en fixe : l'Opéra, la salle Gaveau et Chaillot où je ne suis allé qu'une fois. Il y avait des câbles installés à vie, à Chaillot. Il y en avait bien une quinzaine. Mais la scène était tour à tour utilisée en grande ouverture ou non, alors nous devions réadapter les places des micros. L'intérêt des salles équipées c'est qu'on avait une loge avec une vitre, comme un studio, on pouvait donc écouter sur des enceintes sans gêner le public.

MVD : À cette époque est-ce que les théâtres faisaient leurs propres archivages ?

AL : Il ne faut pas croire que tous les théâtres enregistraient, seulement ceux qui avaient de l'argent! Nous ne savions pas forcément quand ils le faisaient. Une fois ou deux je me suis trouvé en parallèle avec des camarades du théâtre. Ils avaient leurs micros, mais parfois on utilisait les mêmes pour éviter qu'il y en ait trop. Et puis ils n'avaient pas les mêmes tables que nous, qui avions des Telefunken je crois, et ils avaient des disques pas comme les nôtres, avec un trou au centre et un autre sur le côté.

DD : La bande magnétique est arrivée quand chez vous?

AL: En 52 environ. Elle existait bien avant mais elle n'était pas utilisée dans les radios. Entre le disque et l'arrivée des magnétophones il y a eu l'enregistreur Philips-Miller. C'était une bande photographique sur laquelle il y avait une projection de poudre de carbone. Cette bande défilait devant un burin sous un angle d'un degré. Quand le burin s'avançait il enlevait plus ou moins de noir selon la fréquence du son. C'était une gravure mécanique et une lecture par cellule photoélectrique comme pour le cinéma. C'était un très bon enregistreur. L'angle 
était si précis qu'on était obligé d'usiner les pièces sur l'appareil, si bien qu'il n'y avait aucune distorsion de transport. Il y en avait un au Club d'Essai. On a enregistré de très nombreuses dramatiques à la radio avec cet appareil. L'intérêt c'est que l'on pouvait réaliser des montages. Quand les premiers magnétophones sont arrivés, on était encore sur disques et on a travaillé encore longtemps sur disques, même après le magnétophone. Il y avait des magnétos Belin portatifs assez lourds et des fixes, des Tolana. Nagra est arrivé un peu après, ils avaient eu l'intelligence de faire en sorte que la partie mécanique soit à manivelle, parce que le transport de bande demande beaucoup d'énergie, ça a été un miracle, le Nagra, et c'est resté pendant longtemps le meilleur magnétophone au monde.

Dans les salles équipées où l'on avait un matériel fixe, comme à Chaillot, il y avait des lignes PTT de transmission en direct. À l'Opéra il y en a eu très tôt, elles étaient reliées au centre de modulation qui se trouvait rue Armand Moisant et qui renvoyait sur les émetteurs.

\section{L'archivage des spectacles dans les années 1970-1980. L'exemple du TNS}

16 J'ai demandé à Raymond Burger qui, selon lui, aurait été le premier régisseur son en France, de nous parler de ses activités en tant que permanent du théâtre national de Strasbourg, durant quarante années ${ }^{7}$. Il évoque d'abord les conditions d'acquisition de son équipement. Je le cite ici longuement en raison du caractère exceptionnel de ce document, car il n'existe pas d'archive concernant l'évolution des régies.

\section{Matériel Son}

À mon arrivée au Centre dramatique de l'Est, à Strasbourg, en 1963, nous disposions de :

- 2 magnétophones professionnels Belin, 3 magnétophones grand public Philips ;

- 4 amplis mélangeurs à lampes Philips, 10 enceintes colonnes Philips ;

- 4 amplis mélangeurs Bouyer, 8 enceintes Bouyer ;

- 1 console de mixage Philips 6 entrées 2 sorties ;

ce qui était déjà pas mal comme équipement à l'époque.

Au fil des ans, nous avons pu étoffer notre parc. Une fois par an, le ministère nous dotait de matériel. Le matériel son était souvent du Philips. Pratiquement toutes les maisons de la culture entre 1967 et 1970 étaient pourvues en matériel Philips. C'est le ministère qui décidait du choix de matériel.

En 1968, sous André Malraux, et à la demande du directeur, Hubert Gignoux, le ministère nous a nommé théâtre national. Le seul théâtre national de province, totalement subventionné par l'État contrairement à quelques autres, notamment Lyon, qui portait aussi ce titre.

Grâce à la ténacité du directeur technique, Michel Veilhan, [...] j'ai changé d'affectation, de la lumière je suis passé au son - il n'y avait alors pas de service Son. Avec lui, nous avons visité toutes les maisons de la culture équipées et nous avons rédigé un cahier des charges concernant l'équipement du futur théâtre national. Nous avions deux ans avant de prendre possession de notre nouvel outil. En effet le Théâtre de Comédie auquel étaient attachés le Centre dramatique de l'Est et l'École allait changer de bail ; cela permettait à l'État de faire des gros travaux plateau, son et lumière.

Pour la première fois en France, nous avons obtenu la possibilité de pouvoir choisir un matériel plus adapté à nos besoins en passant par un cahier des charges que nous avions proposé, suite à nos visites des théâtres subventionnés, avec soumission et ouverture des plis comme cela se fait pour les marchés d'État. 
Pendant une saison, nous avons pu visiter beaucoup d'installations Son de théâtres, visiter des usines: Philips, Schlumberger, Cabasse et Elipson pour les enceintes, EMT en Allemagne pour les chambres de réverbération et tant d'autres ${ }^{8}$. En 1970, j'avais à ma disposition un équipement Son théâtre unique en France. Plus de 120 enceintes Elipson dans tout le théâtre et les salles de cours.

Notre console fabriquée sur demande, une copie plus ou moins conforme de la console de Pierre Schaeffer à l'ORTF, permettait de travailler le son et surtout de le spatialiser dans la salle.

Voici quel était le matériel de notre cabine régie :

- 1 console prise de son et diffusion 16 entrées micros/lignes avec 8 généraux et 8 circuits auxiliaires de diffusion ;

- 3 magnétophones professionnels Studer ;

- 2 platines tourne-disque à démarrage instantané EMT ;

- 2 lecteurs enregistreurs professionnels de cassettes ;

- 1 magnétophone pour échos construit sur mesure : 1 tête d'enregistrement et 6 têtes de lecture permettant d'enregistrer un son et de le diffuser 6 fois avec des niveaux individuels différents ;

- 1 magnétophone à bande portable ;

- 35 amplis mono de puissance $120 \mathrm{w}$;

- 2 chambres de réverbération EMT à plaques ;

- une batterie de micros allant du micro à condensateur au micro dynamique ;

- un réseau de câblage de la cabine en fond de salle vers le plateau et les salles de cours.

Au théâtre on a surtout besoin d'une sono de très bonne qualité, genre Hi-Fi. Il est évident que dans les moments de silence, il n'est pas permis d'entendre le moindre souffle des enceintes, ce qui n'est pas le cas des sonos de variétés qui diffusent toujours du son à fort niveau.

\section{Un technicien spécialisé}

À partir du moment où un théâtre était équipé d'un matériel conséquent, il fallait une personne sachant s'en servir. J'ai eu la chance d'être là au bon moment, le théâtre et sa technique m'intéressaient au plus haut point. Je me suis préparé à ce poste en dévorant livres et documentations techniques du son, en assistant à plusieurs prises de son à Radio Alsace qui, tous les dimanches, diffusait un concert avec son orchestre philharmonique. J'ai suivi des stages en acoustique architecturale à l'INA. C'est donc à partir de 1969, quand j'ai été détaché à la recherche de matériel son adapté au théâtre et à la rédaction du cahier des charges avec mon directeur technique, que le TNS a créé le poste de régisseur son. Il paraîtrait que j'ai été le premier régisseur son au théâtre...

$\mathrm{Au}$ tout début, que ce soit pour le son ou la lumière, c'était le metteur en scène qui décidait de tout, la nature du son, le niveau, la spatialisation, si on disposait du matériel pour le faire. Très souvent, je l'ai constaté en tournée, on avait mis en place un régisseur son sans qualification spécifique, les metteurs en scène ou directeurs techniques pensant que du moment qu'on avait une chaîne audio ou une cassette chez soi, on pouvait maitriser l'installation sonore.

$\mathrm{Au}$ TNS, le régisseur son participait activement à la création de la pièce. J'ai fait le recensement des « conceptions sonores » que j'ai faites, j'en suis à plus de 40 durant ma carrière ainsi que 11 collaborations aux éclairages. [...] Pour moi le régisseur est polyvalent, avec possibilité d'une attirance particulière pour une spécialité : son, lumière ou plateau, qu'il aura tout loisir d'approfondir tout au long de sa carrière. Petit à petit, il a fallu convaincre les metteurs en scène en proposant des effets ou des ambiances sonores qui pouvaient amener un plus à la perception de la scène. Cela a mis un peu de temps, certains metteurs en scène se sentaient démunis ou 
dépossédés. Mais rapidement ils ont compris ce que la technique pouvait leur apporter. Au TNS la spatialisation sonore a toujours été de mise. Avec l'équipement du théâtre cela devenait obligatoire. [...]

Il n'existait pas de diplôme de créateur, et malgré cela j'ai réussi à former Alain Gravier, chef de service Son à Nanterre, ou le concepteur Michel Maurer, qui enseigne à l'ENSATT de Lyon et au TNS [...] $]^{9}$.

Le témoignage de Raymond Burger est précieux, il rend compte des conditions d'acquisition de l'équipement d'un théâtre national mais, plus encore, il dit l'engagement et l'exigence d'un régisseur devenu concepteur, en faveur du son. L'évolution matérielle montre clairement une qualité d'outils de valeur au moins égale et parfois supérieure à ceux existant au Théâtre de Chaillot. C'est dans ce contexte large qu'il faut inscrire l'archivage des spectacles par le régisseur.

Pour l'archivage des spectacles, des années 1970 à 1981, j'utilisais un enregistreur Studer A62, 1/4 de pouce, bipiste tournant en $19 \mathrm{~cm} / \mathrm{s}$.

Dans la grande salle, les micros étaient placés au milieu de la salle, en bordure du balcon, pratiquement à hauteur d'homme sur scène. L'arc de cercle du balcon a un rayon d'environ $15 \mathrm{~m}$ jusqu'au plateau. Dans la première configuration, j'employais un couple de micros Beyer dynamique M88. Puis, de 1980 à 1995, on enregistrait sur un magnétophone Studer A812 bi-piste 1/4 de pouce, en $19 \mathrm{~cm} / \mathrm{s}$, ou K7 Studer A721 Dolby ou un graveur de CD Studer D741.

Je suis passé assez vite en salle à l'emploi d'un couple coïncidant de micros statiques cardioïdes AKG C451 pour éliminer tout problème de phase dans un passage en mono.

Ce système alimentait aussi l'écoute de scène en cabines son et lumière, ainsi que le retour dans les loges des comédiens et également certains bureaux, tout le monde en profitait.

J'évitais d'utiliser un limiteur compresseur pour bien «sentir» les nuances des niveaux sonores.

Un théâtre est dans l'obligation de pouvoir diffuser les bandes-son des spectacles invités. Il faut donc penser l'équipement en fonction des fiches techniques de nos créations et de nos accueils. C'est dans les années 1980 qu'on a, au TNS, commencé à s'équiper d'enregistreurs CD/DVD professionnels Studer et minidiscs. Nous n'avions pas d'entreprise spécifique pour acheter le matériel son; on choisissait aussi bien le matériel français Schlumberger qu'anglais (pour les amplis Bryston) ou allemand (EMT) et suisse (Nagra, Studer ou Revox).

Ce modèle d'équipement est spécifique aux théâtres nationaux. Pour revenir sur notre choix de mise côte à côte, dans le projet $\mathrm{ECHO}$, des productions archivées du Théâtre de l'Athénée et du Théâtre de Chaillot, les outils et les pratiques décrits par Raymond Burger sont bien différents des outils et pratiques observables durant les mêmes années au Théâtre de l'Athénée, théâtre privé jusqu'en 1982, date à laquelle Pierre Bergé en offrit la tutelle au ministère de la Culture de Jack Lang.

En 1982, j'étais à l'Athénée pour la conception sonore de Platonov mis en scène par Daniel Mesguich, je peux donc témoigner que j'ai travaillé dans un théâtre très mal équipé, mais ni plus ni moins que les autres théâtres privés : un vieux jeu d'orgue à manivelle Sosten à une seule préparation pour la lumière et qui occupait les deux tiers d'une cabine très étroite, commune avec le son. La régie son était très sommaire et l'Athénée avait dû louer du matériel pour ce spectacle exigeant. Peu de haut-parleurs étaient disponibles dans ce théâtre, au point que j'ai dû amener mes propres enceintes. Le réseau électrique mal câblé et non dissocié de celui de la lumière induisait des nuisances sonores dans tous les circuits son. Le régisseur son du lieu, qui n'avait pas été formé, faisait ce qu'il pouvait, et c'est mon assistante, Patricia Delasalle, qui envoyait 
les trois cents effets de son que Mesguich modifiait partiellement chaque soir... L'archivage était effectué par le régisseur son sur un magnétophone amateur à $\mathrm{K} 7$ et les micros qui l'alimentaient étaient écartelés de chaque côté de la scène, dans les loges situées au ras du plateau. La stéréo s'y réduisait à deux monophonies. Voilà les différentes réalités de l'exercice du son théâtral dans une même époque.

\section{L'archivage des spectacles au Théâtre de Chaillot dans les années 1970-1980}

Le 4 septembre 2016, je rencontrai à la Comédie-Française Didier Monfajon qui fut directeur technique durant près de trente années du Théâtre du Palais de Chaillot. Il me fit l'inventaire du dispositif technique qu'il avait trouvé à son arrivée en 1978.

Didier Monfajon: À l'époque il y avait en cabine son de gros magnétophones Schlumberger et une console de la même marque. Ils devaient dater de la réfection de la salle en 1972-74 et être issus des appels d'offre des marchés publics. On changeait les têtes qui s'usaient. Il y avait un responsable des achats qui voulait écouter les bandes pour comprendre pourquoi on voulait les changer puisqu'on entendait encore quelque chose... On a rapidement passé les achats en direction technique en 80-81. Quand je suis arrivé, j'étais régisseur de scène, et je suis rapidement devenu régisseur général à la nomination d'Antoine Vitez avec qui j'avais travaillé au Studio des quartiers d'Ivry.

Daniel Deshays : Tu avais été formé où?

DM : J'avais fait un an et demi à la rue Blanche en Régie administration. En 74, il n'y avait ni son ni lumière dans cette école, il n'y avait que des électriciens, on faisait de l'assistanat de mise en scène, de la régie, du relevé de scène. Il y avait un peu de matériel son mais pas de cours de son. Le directeur m'a proposé un poste au Théâtre de Poche-Montparnasse, j'y suis parti, je suis rentré à Chaillot en 78 comme régisseur de scène, le Poche ayant fermé.

DD : Il faisait quoi le régisseur de scène à cette époque?

DM : On était en répétition quand il y en avait, parce qu'à l'époque il n'y avait plus d'aides à la création et avec l'argent des galas on faisait une ou deux créations par an. J'ai pris un congé sans solde et suis parti à Ivry. De retour à Chaillot, après un an de régie générale, je suis passé directeur technique en 82 .

DD : Y avait-il déjà des régisseurs son?

DM : Oui, ils étaient trois : un en salle Gémier et deux dans la grande salle. Il y avait Jacques Fayoux qui venait de l'Olympia. Avec Vitez, leur nombre a augmenté vu la quantité de productions que l'on faisait. On a dû rapidement quitter la cabine son pour se mettre en salle et donc changer de matériel : on utilisait des Revox, une console Soundcraft 1000 et des enceintes JBL. Il y avait encore des amplis qui fonctionnaient en $100 \mathrm{~V}$ et des Voix du Théâtre : des haut-parleurs qui servaient pour le cinéma et qui étaient accrochés sur une porteuse au fond du plateau. On a dû acheter des Bose 802 pour les placer dans le décor, il n'y avait pas énormément d'enceintes. On a fait construire une console pour Gémier par la Sonétec ; à l'époque il y avait encore des marchés avec le ministère de la Culture. En 83-84 on est devenu maître d'œuvre et on a passé commande directement à Saje pour avoir leur première console numérique, la Memory. C'était la deuxième vendue en France, après Bobigny et avant l'Opéra. Elle était développée avec le système Daisy de chez Fougerolle qui faisait un bruit fou de ventilation et qui avait des disques durs de 
quelques mégas, on pouvait enregistrer 50 secondes ou 1 minute de son ! J'ai dit : on ne va pas s'embarquer là-dedans, et j'ai gardé nos magnétophones. La console coûtait plus d'un million de francs. On l'a séparée en deux et on a mis tous les racks ventilés ailleurs que dans la cabine pour ne garder que la télécommande afin de pouvoir entendre ce qui se passait en scène... On a changé ensuite avec Savary en 88, car lui faisait plutôt du show-business. On est donc repassé sur une console manuelle Yamaha DM1000, puis on en a acheté deux parce que c'était plus facile pour travailler.

DD : Il y avait des créations de bandes?

DM : Non. Avec Savary tout était en direct, c'était surtout de la sono, de l'amplification des voix des comédiens.

DD : Avec Vitez et Aperghis, il y avait de la musique de scène?

DM : Non, pas tant que ça, parce que les Hugo [Hernani et Lucrèce Borgia, 1985], c'était de la création de bandes et avec Aperghis c'était de la musique enregistrée en studio. En direct, on n'a fait que le Monteverdi [Orfeo, 1982], avec un orchestre en scène à Gémier. Sur Le Soulier de satin [1987] on avait un harmonium. On lui avait motorisé les pédales. Il fallait trouver la bonne vitesse pour ne pas trop gonfler le soufflet ! Il avait fait toute la tournée et on n'employait pas d'amplification pour lui.

DD : Vous faisiez l'archivage de tous les spectacles?

DM : Pas systématiquement. Il y a eu l'archivage des bandes de spectacle. Mais la captation, ça n'était pas forcément suivi. J'ai commencé l'archivage vidéo en 1979 sur un spectacle de Victor Garcia avec une caméra N\&B. On a dû commencer à enregistrer les spectacles d'Antoine [Vitez] l'année après Faust, en 1982, et après, c'était selon les moyens financiers dont on disposait, mais ça se faisait en "démonté »: on amenait les caméras qu'on louait le soir et on les rendait vers cinq heures du matin. Nous n'avons pu louer un car régie vidéo qu'en 2000. Une partie des archives sonores d'Antoine est allée à la $\mathrm{BnF}$ dans les années 90 et pour Jérôme [Savary] on ne l'a pas fait.

DD : La prise de son d'archivage était faite comment ? Où se trouvaient les micros?

DM : Ils étaient en fond de salle, à une distance infernale. Après on les a placés de chaque côté du cadre de scène sur des passerelles. Des micros canon. Pour Savary, on avait des PZM placés au sol sur des mousses pour reprendre les voix en comédie musicale. Mais pas de HF. On a eu juste des HF à main sur Ubu [1985] puis on a acheté des micros cravate et des émetteurs Sennheiser Diversity vers 87-88.

DD : Tu as quitté Chaillot en quelle année?

DM : En 2008, après presque trente années !

\section{Épilogue}

21 En 1988, l'occasion m'amena au Théâtre des Bouffes-Parisiens, un petit théâtre privé dirigé alors par Jean-Claude Brialy. J'y réalisais la bande-son, au demeurant assez fournie, de la pièce de Loleh Bellon Une absence, mise en scène par Maurice Bénichou. À mon arrivée, le régisseur m'accueillit et me fit découvrir l'espace qui tenait lieu de régie. Nous pénétrâmes sur scène et il me guida rapidement vers un recoin du plateau 
abrité de la vue du public. Au jardin, derrière le cadre de scène, se trouvait accroché au mur un moniteur vidéo dont l'écran noir et blanc, qui ne dépassait guère une vingtaine de centimètres, lui permettait d'entrevoir la scène de face. Son travail consistait à réaliser la mise en place des accessoires, à la modifier entre chaque scène, à préparer les lumières et à lancer leurs effets, à prendre les notes de répétition dans le cahier de régie et, accessoirement, équipé d'un seul magnétophone, à envoyer les effets son qui d'ordinaire n'étaient guère nombreux... C'était la première fois que je découvrais ce que le théâtre possédait de plus caché et qui remontait du passé.

Je parvins après de longues et houleuses tractations à occuper une loge frontale pour y installer une console et trois magnétophones. Le régisseur fut détaché pour m'assister. Un régisseur lumière, engagé pour la circonstance, resta au plateau. Nous occupions des places dédiées au public, on me fit remarquer le manque à gagner et mon contrat fut modifié en conséquence. Le jour de la Générale, Barbara et Depardieu, présents dans la salle (ils chantaient alors ensemble Lily Passion), voulurent me saluer pour me féliciter du travail sonore accompli, je leur demandai alors d'en remercier surtout la direction!

Ce souvenir nous ramène au début de l'histoire que j'ai entrepris de reconstituer, une histoire qui n'a pas été écrite, dont l'archivage exige, en plus d'un micro convenable, la capacité d'écouter, techniquement mais aussi artisanalement et artistiquement, les professionnels du son. Il s'agit d'une tâche urgente et nécessaire. Si nous considérons que la dimension sonore du théâtre a autant d'importance que sa dimension visuelle, l'histoire de la régie et des régisseurs apparaît comme une composante majeure de l'histoire du théâtre moderne.

\section{NOTES}

1. "Un enseignement critique et transversal du son ", in Le Son du théâtre (XIXe-XXI siècle), JeanMarc Larrue et Marie-Madeleine Mervant-Roux (dir.), Paris, CNRS Éditions, 2016, p. 529-537.

2. À défaut d'être déjà une "nouvelle technologie », qu'elle se contentait de préfigurer.

3. Voir Guillaume Trivulce, "Les métiers du son au théâtre en France », in Le Son du théâtre (XIX $\mathrm{XXI}^{e}$ siècle), op. cit., p. 526, et Daniel Deshays, «Un enseignement critique et transversal du son », ibid., p. 532.

4. «Public Address » : un système d'amplification et de distribution sonore électronique par le biais d'un microphone, d'un amplificateur et de haut-parleurs, permettant à une personne de communiquer un message au grand public, par exemple dans des gares ou des stades. Le terme est également utilisé pour les consoles de mixage, amplificateurs et haut-parleurs de qualité moyenne adaptés à la musique mais aussi à la parole.

5. Les microphones électrodynamiques (dits dynamiques) à bobine mobile ou, mieux, à ruban, produisent leur courant par l'action mécanique du son appliquée sur leur membrane; les électrostatiques modulent le courant par la variation d'une borne d'un condensateur (la membrane) chargée électriquement.

6. Ces boules noires devaient être des « Eight-Balls » de Western Electric. 
7. Voici une rapide biographie professionnelle de Raymond Burger. CAP d'électromécanique, CAP de projectionniste, BEP d'électronicien, Cours d'opérateur de prises de vues. Entrée au CDE (Centre dramatique de l'Est) en 1963. Service Éclairage de 1963 à 1968 (participation à 11 créations lumière). Responsable des services Son et Cinéma de 1968 à 1995 (conception de 35 bandes-son). Responsable Formation Régisseurs, École du TNS, de 1995 à fin 2002. Comédien dans la création de Martinelli en 1995. Comédien dans la création de Stéphane Braunschweig en 2002. Retraite en 2003. «Au total ce furent 40 ans de présence active dans ce théâtre. Voilà mon histoire au CDE devenu TNS ».

8. «Je me souviens particulièrement d'une visite chez M. Léon, fabricant des enceintes Elipson, qui m'a fait visiter l'endroit où il les confectionnait. C'était vraiment artisanal, cela se passait dans une maison dans la banlieue de Paris. J'avais en face de moi le concepteur de ces boules qu'il testait une à une sur un qualiscope pour ajuster le filtrage, c'était passionnant. Voir les staffeurs fabriquer! On en avait partout [au TNS] : une ceinture de 32 boules $40 \mathrm{w}$ en staff au plafond (c'étaient les boules de $40 \mathrm{~cm}$ qu'on voyait partout à l'ORTF), 4 grosses $60 \mathrm{w}$ (boules en polyester, $60 \mathrm{~cm}$ ) au centre du plafond. En ceinture aux murs du théâtre et sur les 3 niveaux les mêmes, 8 circuits permettant un son tournant ( 24 boules staff). Au cadre de scène, c'était un modèle fait sur mesure. Imaginez un gros cylindre pour basses, de la hauteur d'un homme, surmonté par deux boules 2 voies avec tweeters, je pense de $80 \mathrm{w}$, aussi en staff. Sur le plateau j'avais des cubes Elipson en bois de $40 \mathrm{w}$. Il faut dire que dans les années 69-70, tous les théâtres subventionnés ou maisons de la culture étaient presque automatiquement fournis en matériel Philips (ce que mon directeur technique Michel Veilhan et moi-même ne voulions pas). Il a fallu ruser. »

9. J'ai dû amputer ce texte de paragraphes qui rendaient compte de la succession de ses incroyables créations et des conditions d'exploitation dans son théâtre et en extérieur, mais aussi de la formation des régisseurs en interne au TNS, dans une école dont les prémices de l'enseignement datent de 1971.

\section{RÉSUMÉS}

Le sonore du spectacle vivant doit être considéré globalement. Témoins uniques des mouvements - gestes, voix, musiques -, issus de la scène ou d'un enregistrement, tous les sons rendent compte des relations qui y sont mises en jeu.

La création de postes de techniciens a répondu à l'arrivée progressive des technologies spécifiques qui ont modifié les pratiques sonores. L'enregistrement des spectacles s'effectuait différemment pour la radio ou pour l'archivage, tant pour les techniques que pour les outils. Dans ce texte, Albert Laracine, pionnier de la stéréophonie, aborde les pratiques d'enregistrement radiophonique depuis les années 1950, tandis que Raymond Burger brosse son parcours de premier régisseur son au TNS, en particulier dans les années 1970-1980; enfin le directeur technique Didier Monfajon relate ce que fut le son du Palais de Chaillot dans les années 1970-1990.

Sound in live performances must be considered from a global perspective. As unique witnesses to movements - gestures, voices, music -, coming from the stage or recordings, all sounds report on the relations put into play therein.

The creation of technicians' jobs was a response to the gradual arrival of specific technologies that changed sound practices. Performances were recorded differently for radio or archival 
purposes, regarding both techniques and tools. In this text, Albert Laracine, a pioneer in stereophonic sound, discusses radio recording practices since the 1950s, while Raymond Burger sketches his career as a chief sound director at the TNS, with an emphasis on the decade from 1970-1980. Finally, the technical director Didier Monfajon describes sound at the Palais de Chaillot during the years 1970-1990.

\section{INDEX}

Keywords : Management, recording, tape recorder, sound, archive, stereo, microphone, loudspeaker

Mots-clés : Régie, enregistrement, magnétophone, sonore, archive, stéréo, micro, haut-parleur

\section{AUTEUR \\ DANIEL DESHAYS}

Daniel Deshays, réalisateur sonore et professeur des universités, dirige des recherches sur le son. Il travaille depuis plus d'une quarantaine d'années le son pour le théâtre (185 pièces), pour la musique (250 disques) et pour le cinéma (101 films, notamment avec Chantal Akerman, François Caillat, Jean-Michel Carré, Henri Colomer, Richard Coppans, Philippe Garrel, Robert Kramer, Yann Lemasson, Tariq Teguia). Il a fondé le département de Conception sonore à l'ENSATT (École nationale supérieure des arts et techniques du théâtre), enseigne à la Fémis, aux Ateliers Varan et dans de nombreux festivals, Masters et programmes de formation professionnelle. Il a aussi enseigné à l'ENSAD, à Sciences-Po et, durant dix années, à l'ENSBA (École nationale supérieure des Beaux-Arts) de Paris. Il est l'auteur de nombreux articles et de trois essais : Pour une écriture du son (2006), Entendre le cinéma (2010) et Sous l'avidité de mon oreille (2018) parus aux Éditions Klincksieck.

Parmi ses publications :

« Le son du théâtre », in ENSATT. L'école théâtre, Besançon, Les Solitaires Intempestifs, 2011, p. 52-53.

«Face à l'idée de synchronisme », Intermédialités, n 19, Montréal, printemps 2012, p. 85-101.

«Sound \& Gesture », in M. Aramaki, O. Derrien, R. Kronland-Martinet et S. Ystad (dir.), Sound, Music \& Motion, Springer, 2013, p. 483-493.

"Du proche au lointain. L'existence discontinue des bruits et sa représentation continue », Art et bruit. Théâtre, magie, cinéma, musique, radio, opéra, performance, ciné-danse, G. Pisano et M.M. Mervant-Roux (dir.), Ligéia. Dossiers sur l'art, n 141-144, Paris, juil.-déc. 2015, p. 88-93. «Le "paysage sonore" : une congélation du vivant? » in J. Motet (dir.), La Forêt sonore. De l'esthétique à l'écologie, Ceyzérieu, Champ Vallon, 2017, p. 7- 22.

Libertés d'écoute. Construire le hors-temps du sonore, Lyon, Hippocampe Éditions, à paraître en 2019. 